\title{
Super rogue wave generation in the linear regime
}

\author{
Cristian Bonatto, ${ }^{1}$ Sandra D. Prado $\odot,{ }^{1}$ Fernando L. Metz, $, 2,3$ Júlio R. Schoffen $\odot,{ }^{1}$ \\ Ricardo R. B. Correia, ${ }^{1}$ and Jandir M. Hickmann ${ }^{1}$ \\ ${ }^{1}$ Instituto de Física, Universidade Federal do Rio Grande do Sul, 91501-970 Porto Alegre, Brazil \\ ${ }^{2}$ Departamento de Física, Universidade Federal de Santa Maria, 97105-900 Santa Maria, Brazil \\ ${ }^{3}$ London Mathematical Laboratory, 14 Buckingham Street, London WC2N 6DF, United Kingdom
}

(Received 11 April 2019; revised 30 September 2019; accepted 8 November 2020; published 30 November 2020)

\begin{abstract}
Extreme or rogue waves are large and unexpected waves appearing with higher probability than predicted by Gaussian statistics. Although their formation is explained by both linear and nonlinear wave propagation, nonlinearity has been considered a necessary ingredient to generate super rogue waves, i.e., an enhanced wave amplification, where the wave amplitudes exceed by far those of ordinary rogue waves. Here we show, experimentally and theoretically, that optical super rogue waves emerge in the simple case of linear light diffraction in one transverse dimension. The underlying physics is a long-range correlation on the random initial phases of the light waves. When subgroups of random phases appear recurrently along the spatial phase distribution, a more ordered phase structure greatly increases the probability of constructive interference to generate super rogue events (non-Gaussian statistics with superlong tails). Our results consist in a significant advance in the understanding of extreme waves formation by linear superposition of random waves, with applications in a large variety of wave systems.
\end{abstract}

DOI: 10.1103/PhysRevE.102.052219

\section{INTRODUCTION}

In the last decade, a tremendous amount of attention has been devoted to the investigation of extreme or rogue waves, i.e., large amplitude waves appearing more often than predicted by the normal distribution [1,2]. Examples in physical systems arise in hydrodynamics [3], optics [4], plasmas [5], acoustics [6], and quantum systems [7].

A central topic in rogue waves studies is the understanding of the main factors that lead to deviations from the normal distribution. A deep comprehension of the mechanisms associated with the emergence of long-tail statistics is useful for the more ambitious task of predicting and controlling the occurrence of unexpected large events. This is of practical interest, since extreme events can be potentially destructive in different contexts, ranging from damage of maritime structures to breakdown of optical and communication systems. Apart from such practical motivations, there is also a fundamental interest in the phenomenon itself, since the emergence of rogue waves is a complex phenomenon that has triggered considerable efforts of an interdisciplinary scientific community to provide a deep understanding of its generation. Generally speaking, scientists are interested in understanding how highly coherent structures can emerge from disordered and small amplitude systems, being, at same time, statistically significant.

In recent years, remarkable advances have been made in this direction, with noteworthy contributions coming from theoretical developments and controlled experiments with electromagnetic and water waves, in linear and nonlinear wave propagation. In linear systems, it has been shown that, when correlation or inhomogeneity is present in the wave field propagation, an increased probability of generating rogue waves is observed [8-17]. In nonlinear systems, a wealth of wave phenomena has been shown to be relevant for the emergence of rogue waves. In this context, modulational instability has received considerable attention [18-24]. Other mechanisms in nonlinear systems include: breathers collision [25], the dynamics of partially coherent waves [26], the directional properties of the waves [27], and the wind force [28], in the case of water waves; weak nonlinearities without modulation instability in ocean waves [29]; a variety of nonlinear scenarios ranging from chaotic and turbulent flows to solitons and breathers collision in optics [30-46].

Rogue waves are usually defined as waves with more than two times the significant wave height, or exceeding the average amplitude or intensity by four to eight standard deviations $[36,47]$. More recently, some attention has been directed to the investigation of the possibility of generating extremely large amplitude waves, i.e., super localized structures that significantly exceed the usual criteria defining rogue waves. Examples of these so-called super rogue or superextreme waves have been shown experimentally and theoretically in water waves $[48,49]$ and theoretically in a $\mathrm{CO}_{2}$ laser under harmonic modulation [50].

An important open problem is whether nonlinearity is a necessary ingredient to generate such super rogue waves exhibiting an enhanced focusing behavior. The current development stage of rogue waves formation suggests that linear effects can generate only an initial wave amplification, while nonlinearities are responsible for extra wave amplification or focusing, leading to much higher wave amplitudes 
(a)
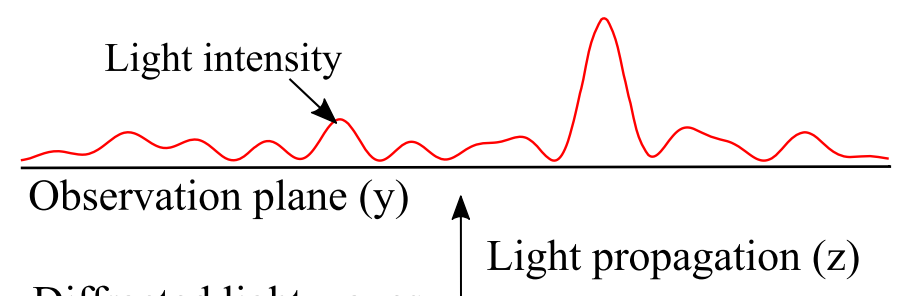

Diffracted light waves

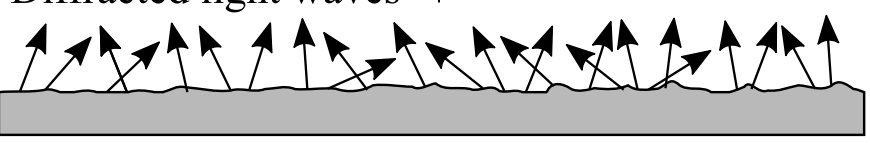

(d)

Uncorrelated phases (b)

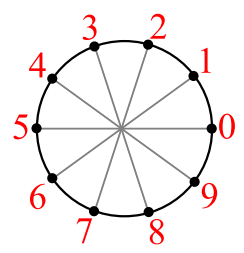

(c)

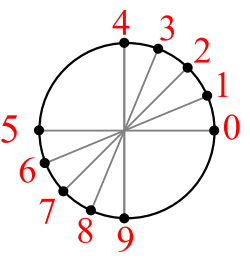

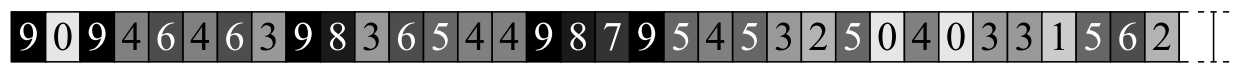

Incommensurate case

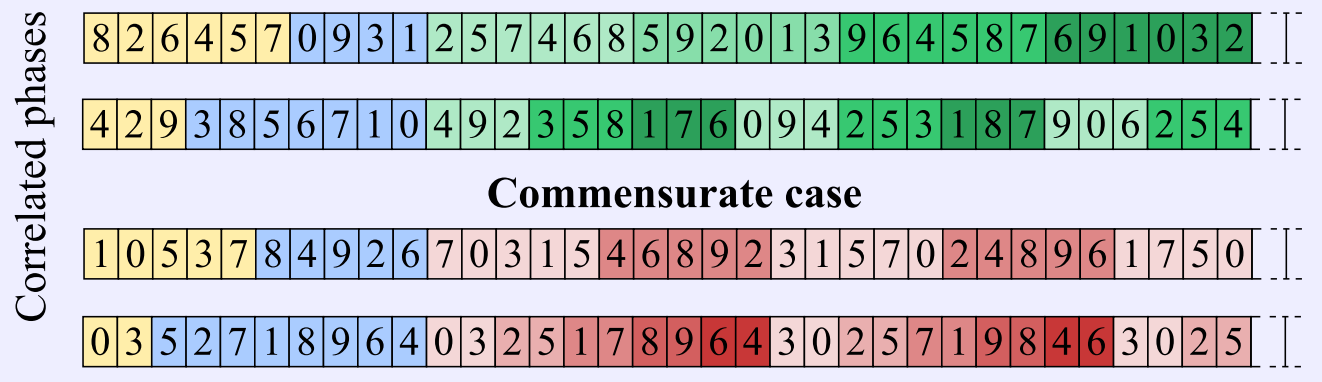

FIG. 1. (a) Illustration of the one-dimensional wave diffraction. (b) and (c) Examples of distinct sets of $L=10$ initial phases, represented by the integers 0 to 9 . (d) Illustration of uncorrelated and correlated initial random phases, corresponding to memoryless, $M=4, M=7$, $M=5$, and $M=8$, from top to bottom. Each integer corresponds to a phase, as shown in (b) or (c). See text for details.

than those observed in the purely linear case [9,18,51-53]. Therefore, a relevant question is whether super localized structures with extremely large amplitudes can emerge from a small-amplitude random field by a purely linear interference, exhibiting, at the same time, a significant probability of occurrence.

In this article, we provide a positive answer to the question above by demonstrating experimentally and numerically the generation of super rogue waves by the simple linear superposition of random waves in an optical system. By studying spatial memory effects of diffracted light waves, we provide the underlying physics behind super rogue waves formation in the linear regime. We show that, when disordered phases exhibit long-range correlations in space, the so-called linear interference model generates waves with amplitudes as high as those observed in systems with strong nonlinearities. It is remarkable that, long after Lord Rayleigh developed his famous statistics when investigating random superposition of sound waves [54], which forms the basis of the so-called Gaussian model for the surface elevation of water waves [55], and after the recent progresses of rogue waves formation in linear systems [8-11,13-15], we can still find a relevant qualitative dynamical scenario in the linear interference model.

\section{MARKOVIAN AND NON-MARKOVIAN INPUT PHASE SEQUENCES}

We consider the simple physical situation of linear and onedimensional light diffraction of a certain number of random waves, as illustrated in Fig. 1(a). Initially, the waves present equal amplitudes and uniformly distributed random phases, with distinct degrees of correlation. After the waves propagate in free space, a diffraction pattern is observed in the far field (Fraunhofer plane). We consider an even number $L$ of distinct initial phases in the interval $[0,2 \pi)$ such that the overall sum of the phasors is zero. The $L$ phases can be equally spaced in the unit circle (spanning all the four quadrants) [Fig. 1(b)] or have another phase configuration, such as $L / 2$ phases in the first quadrant and $L / 2$ phases in the third quadrant [Fig. 1(c)]. Here, we choose the latter case with $L=10$ [56].

Without any correlation, the spatially distributed initial random phases are statistically independent and we obtain a Markovian (memoryless) phase sequence. This uncorrelated case (UC) is illustrated by the gray shade sequence in Fig. 1(d). To generate correlated random phases, we use a variation of a two-dimensional scheme of input phases proposed to investigate diffraction patterns of non-Markovian light [57] and recently used to investigate rogue waves generation in linear and nonlinear media [58]. We start a correlated phase sequence by taking a random permutation of the $L$ distinct phases. The last $M$ random phases, shown in blue color in Fig. 1(d), define the spatial memory length. Then, we perform a random permutation of the remaining $L-M$ phases [shown in yellow color in Fig. 1(d)] and place them to the right of the $M$-phase block. We now identify the last $M$ phases of the formed sequence and repeat successively the previously explained procedure until the total number $N$ of random waves is reached. In this way, a group of $L-M$ random phases 
(a)
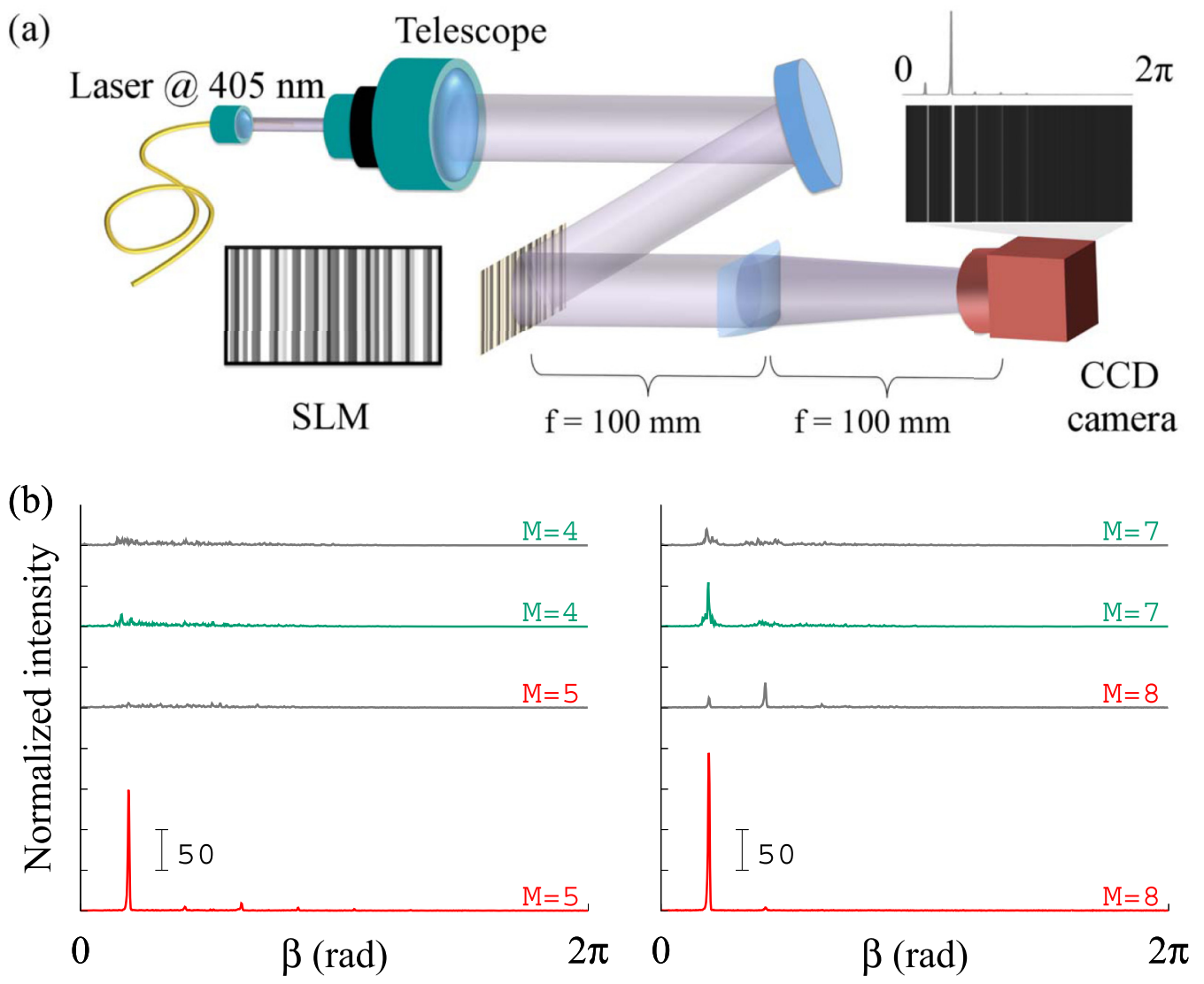

FIG. 2. (a) Experimental setup and illustrations of random input phases and a measured diffraction pattern. (b) Measured diffraction patterns showing the highest measured intensity peak for each corresponding memory length, for the IC (green) and CC (red). Gray plots show the diffraction patterns containing the most frequent maximum intensity from a total number of 1000 realizations. The intensity is normalized by the average intensity of each diffraction pattern and $\beta$ is related to the diffraction angle.

always depends on the previous $M$ phases, resulting in a non-Markovian phase sequence.

The sequences of correlated phases with different memory lengths can be classified in two distinct cases, which we denominate here commensurate case (CC) and incommensurate case (IC). The CC occurs when the ratio $L /(L-M)$ is an integer. In this case, the phase sequence contains distinct subsets of phases with no common elements. For example, for $M=5$ there are two distinct subsets of five random phases, and for $M=8$ there are five subsets of two random phases [see Fig. 1(d)]. In the CC, the spatial configuration of initial phases typically forms a quasiperiodic sequence, where the same group of random phases appears recurrently, which greatly enhances the coherence properties of the waves and favors a quasiresonant process. As we show below, this process is crucial to generate super rogue waves.

\section{LINEAR SUPER ROGUE WAVES}

\section{A. Experiment on diffraction of light}

Our experiments are performed in the following way. One-dimensional random phase sequences, generated in a computer according to the procedure described in Sec. II, are imprinted on the spatial light modulator (SLM), which acts as a phase mask. Since the SLM is a two-dimensional structure and we are interested in investigating the simplest case of one-dimensional wave diffraction, the one-dimensional phase sequences are repeated along the rows of the SLM [a sketch of the SLM is shown in Fig. 2(a), where each gray shade represents a phase value]. The SLM is illuminated by a monochromatic light from a laser beam and the diffracted wavefront propagates in free space, passing through a cylindrical lens up to the detection in a CCD camera. Images recorded with the CCD camera exhibit a one-dimensional intensity pattern, where a single diffraction pattern is calculated averaging all rows in the image of each realization [an example of a recorded image and the respective diffraction pattern is shown in Fig. 2(a)]. This process is repeated for a large number of realizations with different random input phases.

The experimental setup is shown in Fig. 2(a), and additional details are as follows. For the diffracted profiles we used a $5.0 \mathrm{~mW}$ fiber-coupled cw Fabry-Perot laser operating at $405 \mathrm{~nm}$ (Thorlabs- MCLS1), whose output is sequentially collimated with a $75 \times$ beam expander. The resulting wave front illuminates the active area of the SLM-1280×720 pixels of $9.5 \mathrm{~mm}$ pitch (Cambridge-SDE1280)—containing the designed spatial phase patterns. The SLM provides, according to an eight-bit gray level, the phase structure randomly generated in a computer. This diffracted wave front illuminates a cylindrical lens with focal distance $f=100 \mathrm{~mm}$, which in turn images the intensity pattern of the diffracted beam 

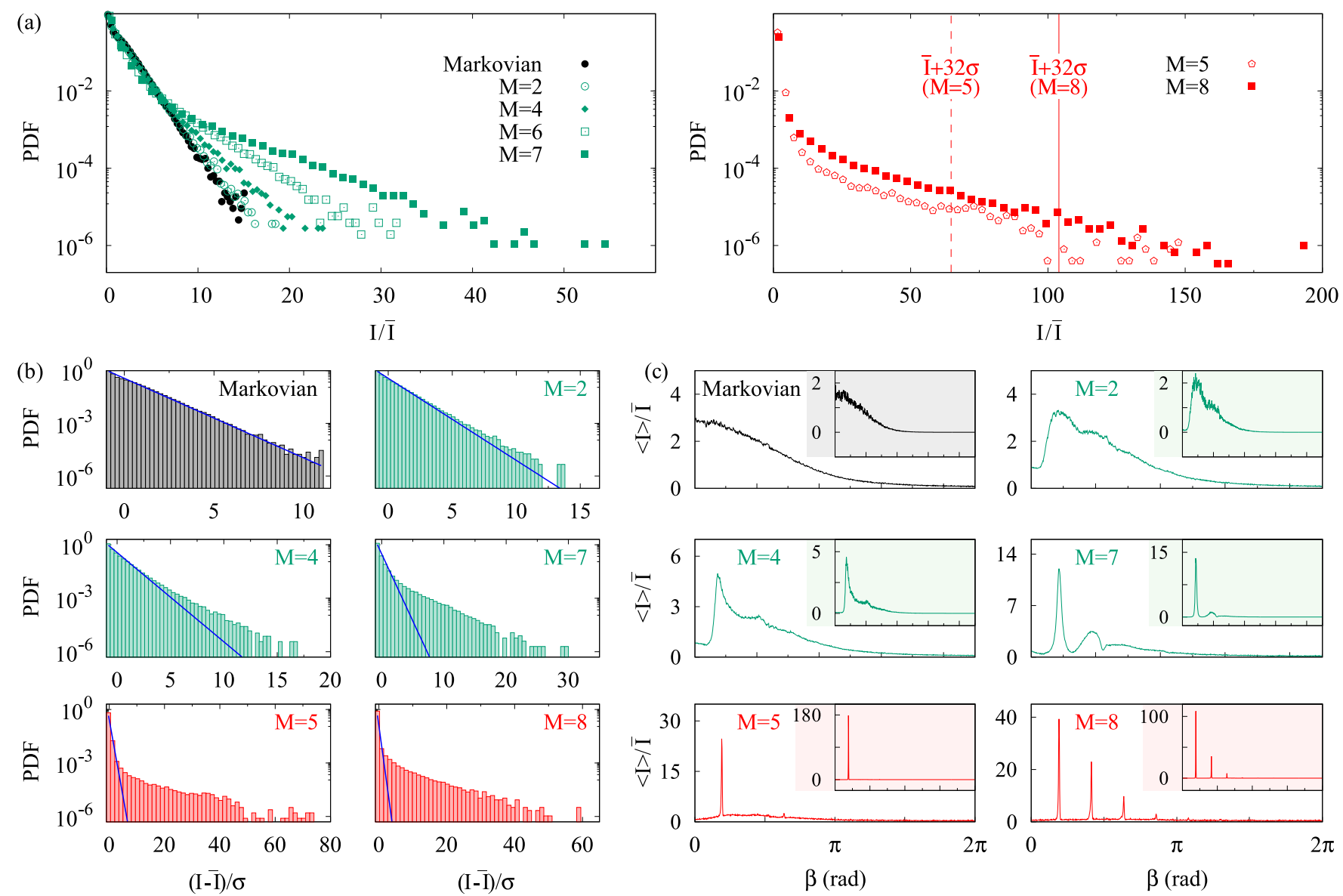

FIG. 3. (a) Probability density function (PDF) of the measured light intensity. The dashed and solid vertical lines mark the super rogue wave definition for the cases $M=5$ and $M=8$, respectively. (b) Left: PDFs of the light intensity normalized by its standard deviation $\sigma$. The blue straight line denotes the negative exponential fitting. Right: Average intensity $\langle I\rangle$ and variances (insets) as a function of screen position $\beta$. The overall average $\bar{I}$ and the standard deviation $\sigma$ are obtained by averaging over all realizations and all points recorded on the camera, for each correlation degree. (c) PDFs from numerical simulations.

onto a CCD camera (Point Grey - CMLN-13S2M-CS) with $1280 \times 960$ pixels of $3.5 \mathrm{~mm}$ pitch. Images excluding the central maxima were registered for a set of 1000 realizations up to the first diffraction order.

The effects of distinct memory lengths on the measured light intensities are shown in Fig. 2(b). The parameter $\beta=$ $k a \sin \theta$ is related to the diffraction angle $\theta$, where $k$ is the magnitude of the wave vector of the incident wave and $a$ is the pixel width of the SLM. Figure 2(b) shows the measured diffraction pattern containing the highest intensity peak for some memory lengths corresponding to the IC (green curves) and $\mathrm{CC}$ (red curves). We can see that the the memory lengths of the CC, $M=5$ and $M=8$, produce much higher light intensity peaks than the memory lengths of the IC, $M=4$ and $M=7$. The gray curves in Fig. 2(b) show typical realizations of single diffraction patterns where the highest intensity peak equals the mode of the statistical distribution of the maxima intensities. In other words, if we take a random realization, the highest intensity peak of the diffraction pattern is more likely to coincide with the highest intensity in the gray curves.

In Fig. 3(a), we show, for distinct correlation degrees, a plot of the probability density function (PDF) of the intensity $I$ normalized by its overall average $\bar{I}$, obtained from all different realizations and all points from the camera. In the UC (black points), the intensity follows a negative exponential distribution, which is a signature of Rayleigh statistics. In this case, the high intensity events have an extremely low probability of occurrence. In the IC (green points), events with higher intensities are more likely to occur than in the UC. As memory increases, deviations from Rayleigh statistics become more pronounced, leading to long-tailed PDFs. This is the usual signature of extreme waves. In the CC (red points), the PDFs exhibit superlong tails, due to what we call super rogue waves. Below we discuss the quantitative and qualitative differences between rogue and super rogue waves. Note that super rogue light intensity events, such as those observed for $M=5$ and $M=8$, are very unlikely to occur in the IC, even when considering $M=7$, a case of large memory length.

A usual criterion to define extreme events is the comparison of the intensity (or amplitude) of an event with the average intensity (or amplitude) plus a certain number of standard deviations $[36,47]$. Throughout this article, we define super rogue waves as those waves with intensities exceeding the overall average intensity by more than 32 standard deviations $\left(I_{\mathrm{SRW}}=\bar{I}+32 \sigma\right)$. By this definition, the theshold is four times greater than that of the $8 \sigma$ criterion [36], a usual 
criterion for defining ordinary rogue waves. This super rogue wave definition has the advantage of being precise and the drawback of being quite arbitrary. Other statistical measures, such as the comparison of the PDFs with a stretched exponential distribution $[35,53]$ or the analysis of the kurtosis instead of the variance [20], could be used to quantify deviations from Gaussianity. Nevertheless, a definition of super rogue waves by these criteria would present some degree of arbitrariness as well. In Fig. 3(b), we plot the PDF of $I-\bar{I}$ normalized by its standard deviation $\sigma$. Thus, the horizontal scales of the PDF plots in Fig. 3(b) indicate directly the number of standard deviations by which the measured intensity exceeds the average intensity. The experimental results show waves with intensities exceeding the average intensity by more than 32 standard deviations in the CC [see the $M=5$ and $M=8$ cases in Fig. 3(b)]. The CC exhibits a number of waves with intensities 100 times greater than the average intensity, as can be seen in the experimental PDFs [Fig. 3(a)], with a much higher probability of occurrence than in the UC and the IC. The results observed for the $\mathrm{CC}$ show a much more significant wave amplification when compared with the case of ordinary rogue waves.

In Fig. 3(c) we show the average and the variance of the light intensity along the screen, obtained from averaging over all diffraction patterns. The light intensity decays as we depart from $\beta=0$, since the pixel size in the SLM produces a $\operatorname{sinc}(\beta / 2)$ modulation with the first diffraction minimum located at $\beta=2 \pi$. The mean intensity of the uncorrelated case approximately reproduces this modulation. For the correlated cases, the average intensity is small at $\beta=0$, which is a consequence of the zero sum of the input phases and their correlation. In this case, there is no repetition between neighboring input phases, since the minimum distance between two equal phases in a sequence is given by the memory length. As the memory increases, there are some specific screen positions where it is more likely to observe extreme events, as can be seen by the peaks in the average and the variance of the light intensity. These peaks are much more pronounced in the CC than in the IC, which is a direct consequence of the more ordered input phases, forming a quasiperiodic sequence.

\section{B. Theoretical simulations}

A simple linear model reproduces qualitatively the experimental results. We model the SLM as a one-dimensional array with a total number of $N$ pixels, each one characterized by a finite width and a random phase. An incident monochromatic wave diffracts on the SLM, propagates in free space, and yields a resultant electric field on the Fraunhofer plane

$$
E=E_{0} \operatorname{sinc}(\beta / 2) \sum_{m=1}^{N} e^{i m \beta+i \phi_{m}}
$$

where $E_{0}$ is the amplitude of the incident electric field and $\phi_{m}$ is the random phase of pixel $m$. For the theoretically computed diffraction patterns, we performed numerically the summation in Eq. (1) by using $E_{0}=1$ and $N=1024$ random waves with the same initial phases used in the experiments and explained in Fig. 1. The resultant electric field on the screen is computed for 20000 values of $\beta$, equally spaced in the interval $[0,2 \pi)$.
The statistical distributions of 1000 realizations for the numerically computed intensity $I=|E|^{2}$ are shown in Fig. 4, for distinct phase correlations. The qualitative agreement between the theoretical results of Fig. 4(a) and the experimental results of Fig. 3(a) is very good. In the simple theoretical model we use here, which qualitatively reproduces the experiments, a number of waves with intensities 300 times greater than the average intensity appear for the CC [Fig. 4(a)]. Such waves exceed by far usual criteria of rogue waves definition, characterizing wave events with superextreme amplitudes. These super rogue events are very unlikely to be found in a random realization of the IC. In Fig. 4(b), we show the average and variance computed for the whole cases of distinct initial phase correlations investigated in the experiments. Again, the overall agreement with the experiments is very good. In Fig. 4(c), we show the PDFs obtained from the numerical simulations by using the initial phases simmetrically distributed along the four quadrants, between 0 and $2 \pi$, according to Fig. 1(b). As we can see, there is not a qualitative difference when compared with the simulations using the initial phases only in the first and third quadrants, which have been used in the experiments.

The main ingredient behind the distinct qualitative behaviors of the UC, IC, and CC is the correlation properties due to memory effects of the initial phases. In order to better understand the role of the memory length in producing coherence, we numerically calculate the following correlation between two spatially separated blocks containing $L$ random phases:

$$
C_{d}=\frac{1}{L} \sum_{i=1}^{L}\left\langle\phi_{i} \phi_{i+d L}\right\rangle,
$$

where $d=1,2,3, \ldots$ is the distance between the first block with $L$ phases to the other subsequent blocks along the phase sequence. In Eq. (2) the product $\phi_{i} \phi_{i+d L}$ is equal to one if $\phi_{i}=\phi_{i+d L}$ and zero otherwise. The angle brackets $\langle\ldots\rangle$ denote the average over 1000 distinct realizations of the random phases. A plot of the correlation as a function of the distance $d$ between the blocks, for fixed $L=10$ and different memory lengths, is shown in Fig. 5(a). In the IC, the correlation decays as the distance between the blocks increases, converging to $1 / L$. This means that, for large distances, the probability of finding two coherent phases is $1 / L$, which is the same value as in the UC. In contrast, the correlation does not decay in the $\mathrm{CC}$, having the constant value $1 /(L-M)$. In other words, the probability of finding two coherent phases separated by an arbitrary number of blocks of size $L$ is 0.2 and 0.5 for $M=5$ and $M=8$, respectively. This is a significant difference when compared with the IC.

The overall coherence of the phase sequence, for each $M$, is obtained by computing the integrated correlation

$$
\bar{C}=\frac{1}{n} \sum_{d=1}^{n}\left(C_{d}-\frac{1}{L}\right)^{2},
$$

where $n$ is the maximum distance used in the calculation. Since each correlation in the IC quickly decays to the value $1 / L$, we used $n=50$, which already provides the asymptotic value of $\bar{C}$. For the $\mathrm{CC}, C_{d}$ is independent of $d$, thus the integrated correlation is independent of $n$. As shown in 

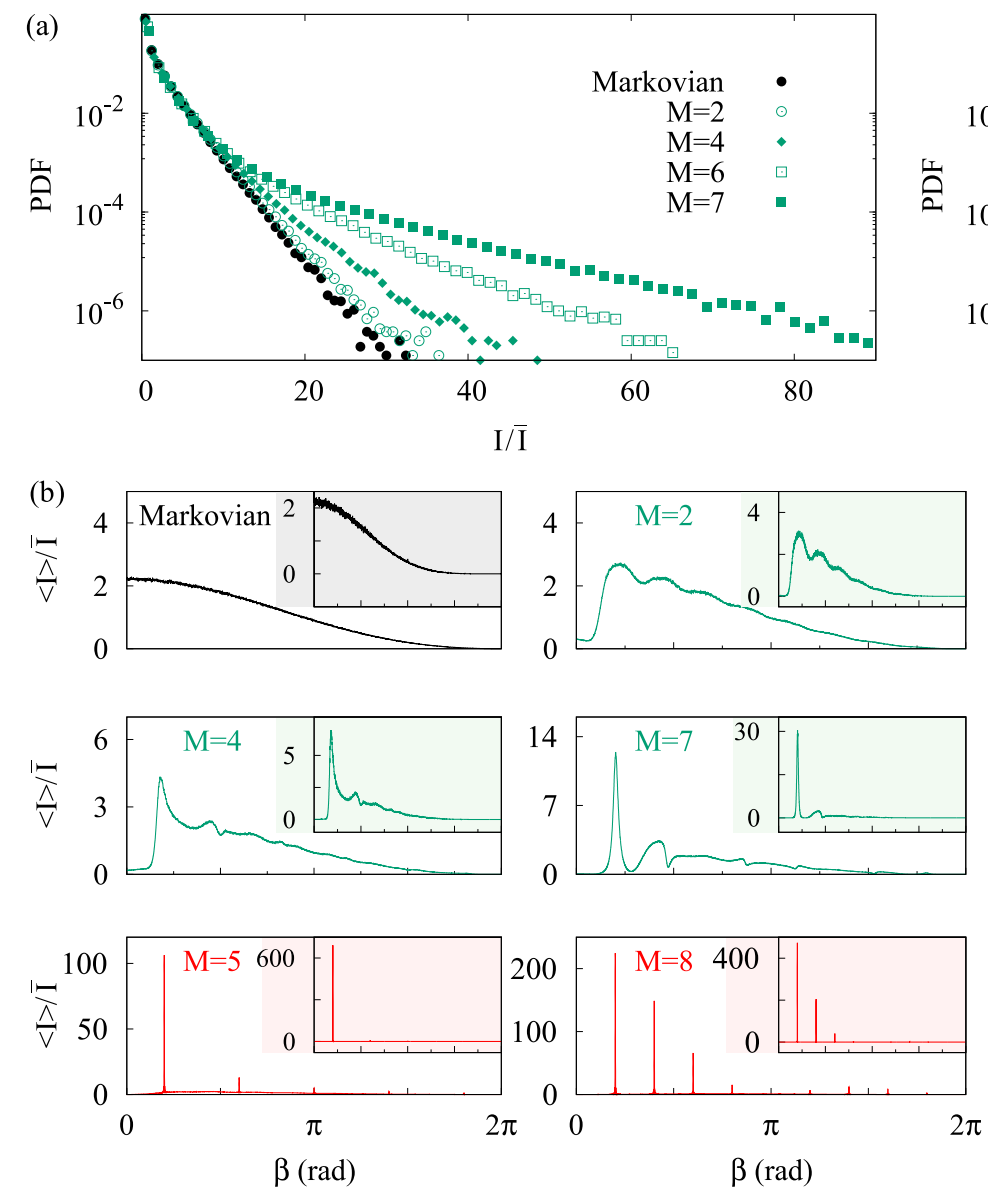
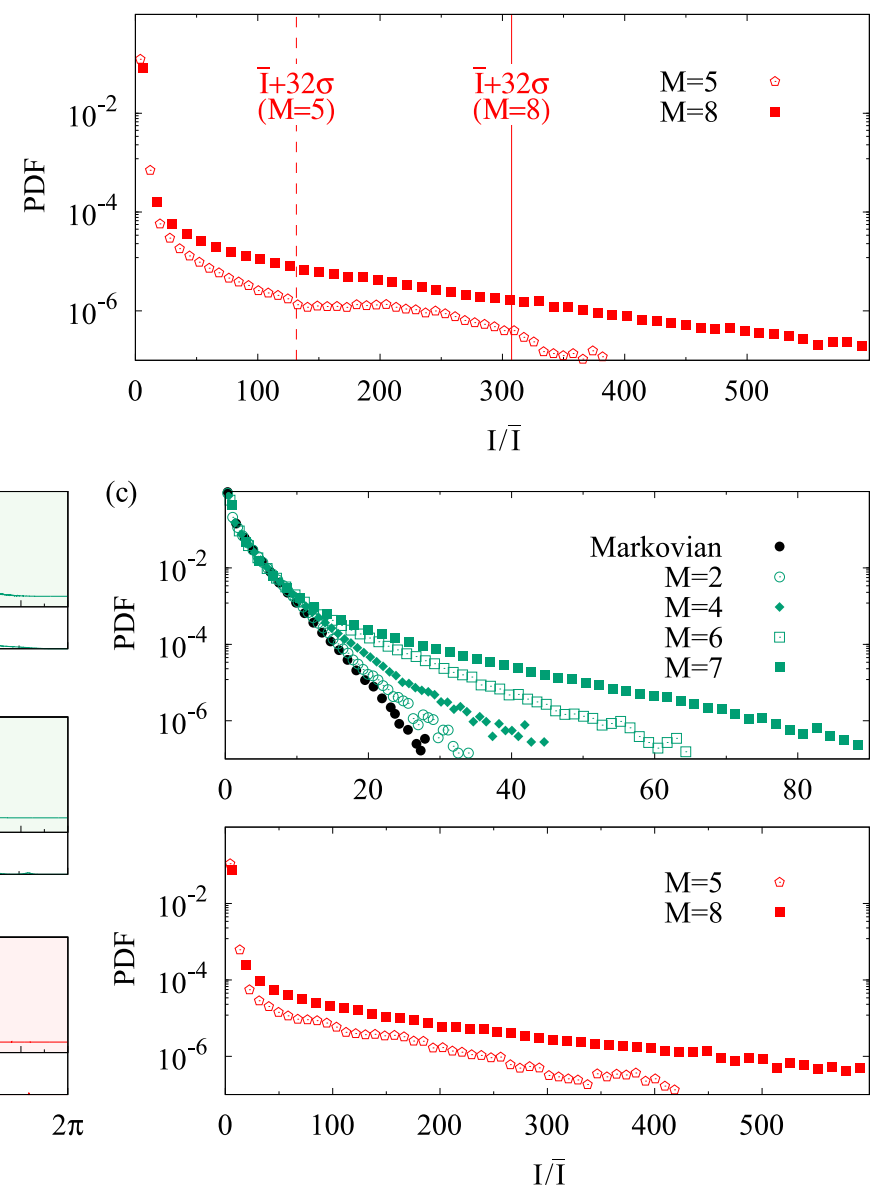

FIG. 4. (a) Probability density function (PDF) of the numerically computed light intensity for the UC and IC (left) and the CC (right). The dashed and solid vertical lines mark the super rogue wave definition for the cases $M=5$ and $M=8$, respectively. The light intensity is normalized by the overall average intensity $\bar{I}$. (b) Average intensity $\langle I\rangle$ as a function of screen position, $\beta$, obtained from all realizations. The insets show the corresponding variances. (c) Numerically computed PDFs with the initial phases distributed along the four quadrants.

Fig. 5(b), the integrated correlation puts in a clear ordering the coherence generated by distinct memory lengths, having $M=5$ and $M=8$ as the most coherent cases. This ordering of the coherence properties of the initial phases directly reflects in the amplitudes of the extreme events generated by wave diffraction. We stress that only a large memory length is not enough for producing strong coherence. As can be seen in Fig. 5(b), the smaller memory length $M=5$ of the CC can generate more coherence than larger memory lengths $M=6$ and $M=7$ of the IC.

Another way to observe the coherence properties of the initial random waves is plotting the Fourier transform of the phase sequences. These results are shown in Fig. 6 and are very similar to the intensity patterns shown in Fig. 4(b), since an intensity pattern observed after the linear wave propagation, in the far field, is computed through a Fourier transform of the initial random waves. The main difference is that the intensity patterns observed after the wave propagation contain a sinc modulation, exhibiting a decaying intensity far from the central position of the screen. A more ordered structure (or a higher degree of periodicity) of the initial phase sequences corresponds to a higher probability of finding extreme events through wave diffraction. As can be seen in
Fig. 6, the spatial phase sequences belonging to the $\mathrm{CC}$ contain a much higher degree of ordering, since they correspond to quasiperiodic phase sequences. This condition significantly increases the probability of finding super rogue waves through wave diffraction. Despite the fact that the $\mathrm{CC}$ can generate super rogue events, an isolated random realization from our method of generating the phase sequences typically does not exhibit some significant degree of periodicity. This is illustrated in Fig. 7(a), where we show a random phase sequence without exhibiting some significant degree of periodicity, for the $M=5$ case (the respective intensity pattern is shown in Fig. 2, by the gray curve). But, for the $\mathrm{CC}$, the probability of generating a high degree of periodicity is very large. The CC contains a very relevant feature: the match between two spatial scales, i.e., the $L$ and $L-M$ sizes. This condition greatly enhances the probability of generating sequences with a very ordered structure, with long-range correlations, similar to what we have in a quasiresonant process. In Fig. 7(b), we illustrate this with a realization for the $M=5$ case where the phase sequence displays a high degree of periodicity. Therefore, super rogue waves can be generated with a significant probability in the $\mathrm{CC}$, while in the UC and IC the probability of finding super rogue waves is not significant. 

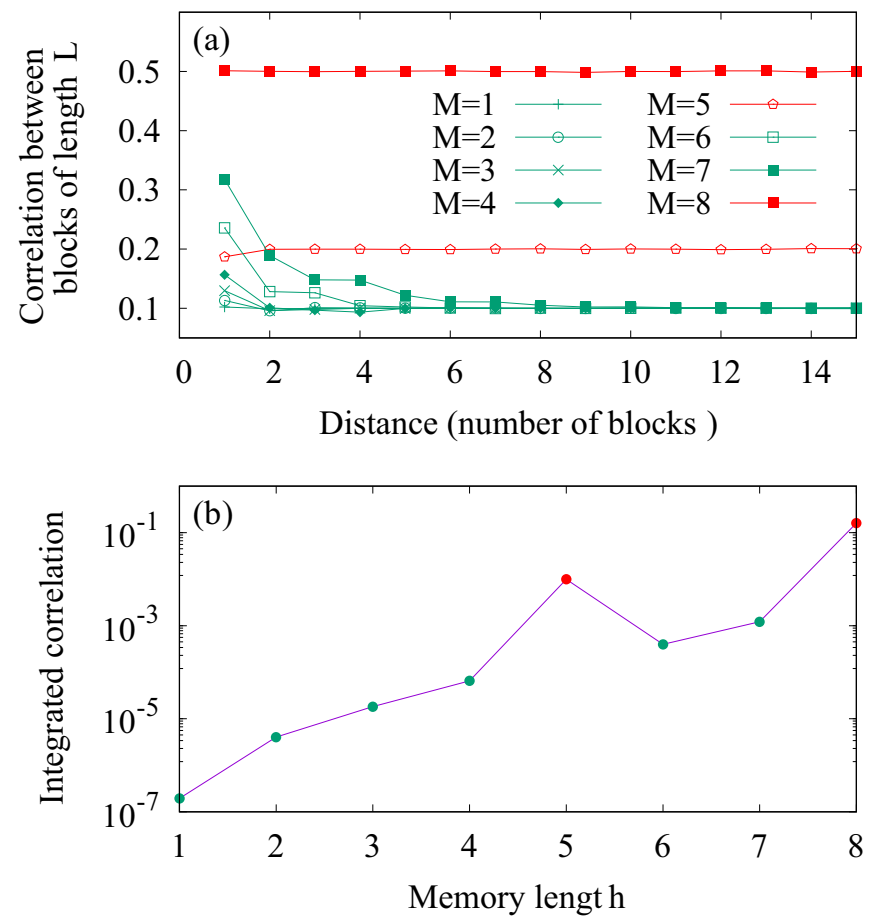

FIG. 5. (a) Correlation between spatially separated pairs of blocks containing $L=10$ random phases as a function of their distance, measured in number of blocks along the phase sequence. The red and green curves denote the $\mathrm{CC}$ and IC, respectively. (b) Integrated correlation as a function of memory length. Each integrated correlation is proportional to the area under the corresponding curve shown in (a).
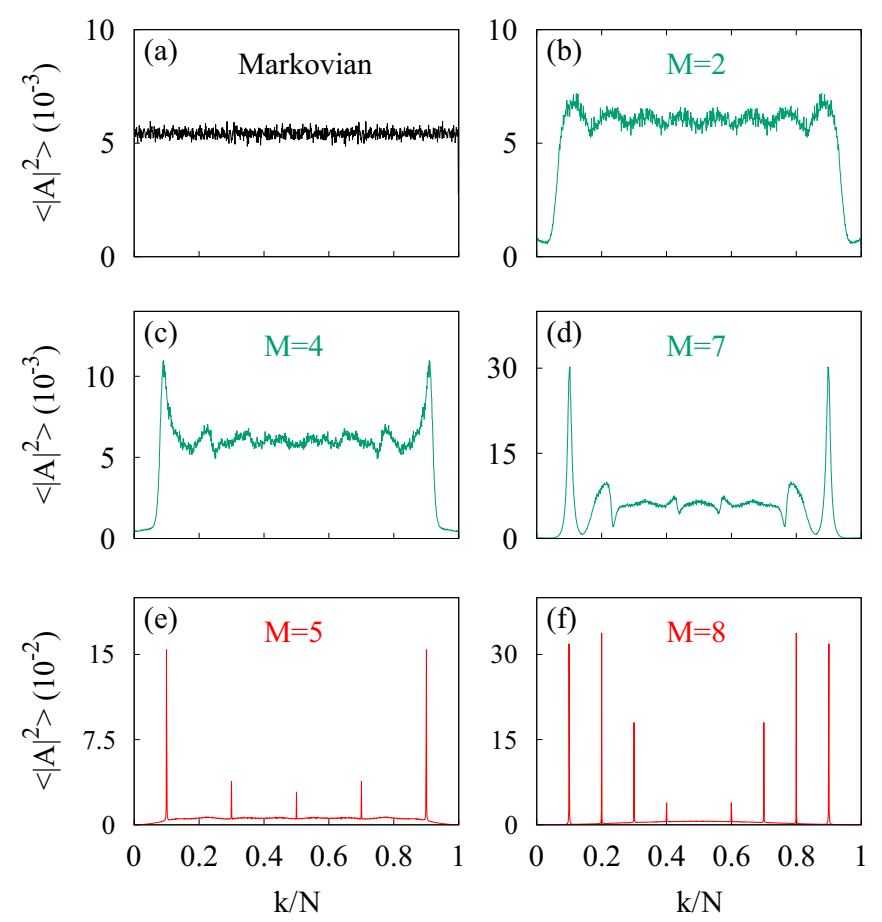

FIG. 6. Average of the Fourier transform of the initial phase sequences computed for 1000 random sequences containing $N=1024$ phases for each memory length. $A$ and $k$ are the amplitude spectrum and the wave number, respectively.
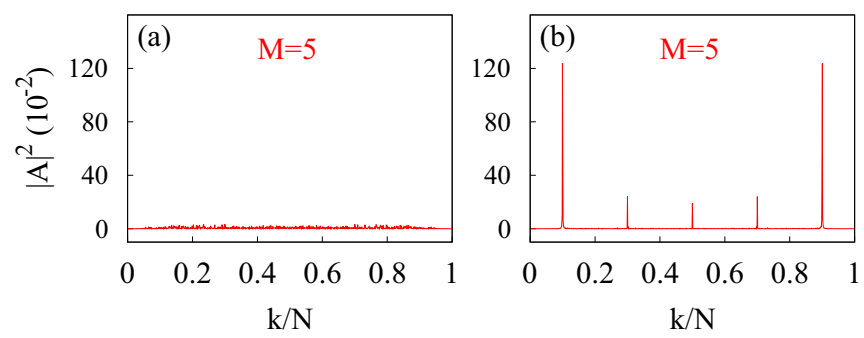

FIG. 7. The Fourier transform of two distinct random phase sequences of the commensurate case $M=5$.

\section{CONCLUSION}

In conclusion, by studying systematically memory effects of spatially distributed random phases of a light field, we have shown that a simple linear superposition of random waves can generate waves with superextreme amplitudes, with a significant probability of occurrence. Up to date, super rogue waves, exceeding by far the usual criteria of rogue waves definition, have been observed, in both hydrodynamical and optical systems, only when nonlinearities are present. The linear super rogue waves investigated here have been generated by using small phase fluctuations between 0 and $2 \pi$ and they are not only quantitatively, but also qualitatively distinct when compared with ordinary rogue waves, since they are formed by long-range phase correlations. Although we use a particular procedure for generating correlations between the optical phases of the interfering waves, other kinds of long-range correlations should yield similar effects. The solutions of the linear interference model can be classified in distinct qualitative groups as a function of the phase correlations in the input waves. In two opposite limits, we have the fully disordered case (uncorrelated phases) and the fully ordered case (completely coherent phases). Random uncorrelated phases lead to the well-known Rayleigh statistics, where high-amplitude waves are very unlikely. Completely coherent phases, as found in elementary physics textbooks, lead to a sinc-like pattern. Between these two limits, we can have two distinct qualitative scenarios: short-range and long-range correlated random phases. Short-range correlated phases lead to an increased probability of generating extreme waves, which is the usual investigated case in the rogue waves literature. On the other hand, long-range correlations of the random phases greatly increase the probability of generating waves with superextreme amplitudes, yielding a much more pronounced L-shaped statistics when compared with ordinary rogue waves.

\section{ACKNOWLEDGMENTS}

C.B. thanks National Council for Scientific and Technological Development-CNPq for financial support under Contract No. 487057/2013-9. F.L.M. thanks London Mathematical Laboratory and CNPq (Edital Universal 406116/2016-4) for financial support. 
[1] M. Onorato, S. Residori, U. Bortolozzo, A. Montina, and F. T. Arecchi, Phys. Rep. 528, 47 (2013).

[2] N. Akhmediev, A. Ankiewicz, and M. Taki, Phys. Lett. A 373, 675 (2009).

[3] C. Kharif, E. Pelinovsky, and A. Slunyaev, Rogue Waves in the Ocean (Springer, Heidelberg, 2009).

[4] D. R. Solli, C. Ropers, P. Koonath, and B. Jalali, Nature (London) 450, 1054 (2007).

[5] H. Bailung, S. K. Sharma, and Y. Nakamura, Phys. Rev. Lett. 107, 255005 (2011).

[6] A. N. Ganshin, V. B. Efimov, G. V. Kolmakov, L. P. MezhovDeglin, and P. V. E. McClintock, Phys. Rev. Lett. 101, 065303 (2008).

[7] Y. V. Bludov, V. V. Konotop, and N. Akhmediev, Phys. Rev. A 80, 033610 (2009).

[8] B. S. White and B. Fornberg, J. Fluid Mech. 355, 113 (1998).

[9] E. J. Heller, L. Kaplan, and A. Dahlen, J. Geophys. Res. 113, C09023 (2008).

[10] R. Höhmann, U. Kuhl, H.-J. Stöckmann, L. Kaplan, and E. J. Heller, Phys. Rev. Lett. 104, 093901 (2010).

[11] F. T. Arecchi, U. Bortolozzo, A. Montina, and S. Residori, Phys. Rev. Lett. 106, 153901 (2011).

[12] S. Vergeles and S. K. Turitsyn, Phys. Rev. A 83, 061801(R) (2011).

[13] J. J. Metzger, R. Fleischmann, and T. Geisel, Phys. Rev. Lett. 112, 203903 (2014).

[14] C. Liu, R. E. C. van der Wel, N. Rotenberg, L. Kuipers, T. F. Krauss, A. Di Falco, and A. Fratalocchi, Nat. Phys. 11, 358 (2015).

[15] A. Mathis, L. Froehly, S. Toenger, F. Dias, G. Genty, and J. M. Dudley, Sci. Rep. 5, 12822 (2015).

[16] S. Derevyanko, A. Redyuk, S. Vergeles, and S. Turitsyn, APL Photon. 3, 060801 (2018).

[17] R. da Silva and S. D. Prado, Phys. Lett. A 384, 126231 (2020).

[18] M. Onorato, A. R. Osborne, M. Serio, and S. Bertone, Phys. Rev. Lett. 86, 5831 (2001).

[19] C. Kharif and E. Pelinovsky, Eur. J. Mech B Fluids 22, 603 (2003).

[20] P. A. E. M. Janssen, J. Phys. Oceanogr. 33, 863 (2003).

[21] D. R. Solli, G. Herink, B. Jalali, and C. Ropers, Nat. Photonics 6, 463 (2012).

[22] A. I. Dyachenko and V. E. Zakharov, J. Exp. Theor. Phys. Lett. 81, 255 (2005).

[23] F. Baronio, M. Conforti, A. Degasperis, S. Lombardo, M. Onorato, and S. Wabnitz, Phys. Rev. Lett. 113, 034101 (2014).

[24] B. Kibler, A. Chabchoub, A. Gelash, N. Akhmediev, and V. E. Zakharov, Phys. Rev. X 5, 041026 (2015).

[25] N. Akhmediev, J. Soto-Crespo, and A. Ankiewicz, Phys. Lett. A 373, 2137 (2009).

[26] R. El Koussaifi, A. Tikan, A. Toffoli, S. Randoux, P. Suret, and M. Onorato, Phys. Rev. E 97, 012208 (2018).

[27] M. Onorato, T. Waseda, A. Toffoli, L. Cavaleri, O. Gramstad, P. A. E. M. Janssen, T. Kinoshita, J. Monbaliu, N. Mori, A. R. Osborne, M. Serio, C. T. Stansberg, H. Tamura, and K. Trulsen, Phys. Rev. Lett. 102, 114502 (2009).

[28] A. Toffoli, D. Proment, H. Salman, J. Monbaliu, F. Frascoli, M. Dafilis, E. Stramignoni, R. Forza, M. Manfrin, and M. Onorato, Phys. Rev. Lett. 118, 144503 (2017).
[29] F. Fedele, J. Brennan, S. Ponce de León, J. Dudley, and F. Dias, Sci. Rep. 6, 27715 (2016).

[30] S. Toenger, T. Godin, C. Billet, F. Dias, M. Erkintalo, G. Genty, and J. M. Dudley, Sci. Rep. 5, 10380 (2015).

[31] P. Walczak, S. Randoux, and P. Suret, Phys. Rev. Lett. 114, 143903 (2015).

[32] K. Hammani, C. Finot, J. M. Dudley, and G. Millot, Opt. Express 16, 16467 (2008).

[33] D. Borlaug, S. Fathpour, and B. Jalali, IEEE Photonics J. 1, 33 (2009).

[34] D. V. Churkin, O. A. Gorbunov, and S. V. Smirnov, Opt. Lett. 36, 3617 (2011).

[35] A. Montina, U. Bortolozzo, S. Residori, and F. T. Arecchi, Phys. Rev. Lett. 103, 173901 (2009).

[36] C. Bonatto, M. Feyereisen, S. Barland, M. Giudici, C. Masoller, Jose R. Rios Leite, and J. R. Tredicce, Phys. Rev. Lett. 107, 053901 (2011).

[37] F. Selmi, S. Coulibaly, Z. Loghmari, I. Sagnes, G. Beaudoin, M. G. Clerc, and S. Barbay, Phys. Rev. Lett. 116, 013901 (2016).

[38] P. Walczak, C. Rimoldi, F. Gustave, L. Columbo, M. Brambilla, F. Prati, G. Tissoni, and S. Barland, Opt. Lett. 42, 3000 (2017).

[39] J. Kasparian, P. Béjot, J.-P. Wolf, and J. M. Dudley, Opt. Express 17, 12070 (2009).

[40] A. N. Pisarchik, R. Jaimes-Reátegui, R. Sevilla-Escoboza, G. Huerta-Cuellar, and M. Taki, Phys. Rev. Lett. 107, 274101 (2011).

[41] C. Lecaplain, P. Grelu, J. M. Soto-Crespo, and N. Akhmediev, Phys. Rev. Lett. 108, 233901 (2012).

[42] V. Odent, M. Taki, and E. Louvergneaux, Nat. Hazards Earth Syst. Sci. 10, 2727 (2010).

[43] A. K. D. Bosco, D. Wolfersberger, and M. Sciamanna, Opt. Lett. 38, 703 (2013).

[44] C. J. Gibson, A. M. Yao, and G.-L. Oppo, Phys. Rev. Lett. 116, 043903 (2016).

[45] D. Pierangeli, F. Di Mei, G. Di Domenico, A. J. Agranat, C. Conti, and E. DelRe, Phys. Rev. Lett. 117, 183902 (2016).

[46] D. Pierangeli, F. Di Mei, C. Conti, A. J. Agranat, and E. DelRe, Phys. Rev. Lett. 115, 093901 (2015).

[47] K. Dysthe, H. E. Krogstad, and P. Müller, Annu. Rev. Fluid Mech. 40, 287 (2008).

[48] A. Chabchoub, N. Hoffmann, M. Onorato, and N. Akhmediev, Phys. Rev. X 2, 011015 (2012).

[49] A. Chabchoub, N. Hoffmann, M. Onorato, A. Slunyaev, A. Sergeeva, E. Pelinovsky, and N. Akhmediev, Phys. Rev. E 86, 056601 (2012).

[50] C. Bonatto and A. Endler, Phys. Rev. E 96, 012216 (2017).

[51] A. I. Dyachenko, D. I. Kachulin, and V. E. Zakharov, Stud. Appl. Math. 137, 189 (2016).

[52] M. Mattheakis, I. J. Pitsios, G. P. Tsironis, and S. Tzortzakis, Chaos, Soliton Fract. 84, 73 (2016).

[53] A. Safari, R. Fickler, M. J. Padgett, and R. W. Boyd, Phys. Rev. Lett. 119, 203901 (2017).

[54] Lord Rayleigh, London, Edinburgh, Dubline Philos. Mag. J. Sci. 10, 73 (1880).

[55] M. S. Longuet-Higgins and G. E. R. Deacon, Philos. Trans. R. Soc. London, Ser.es A 249, 321 (1957).

[56] We have chosen the initial phases distributed only in the first and third quadrants due to experimental limitations, since our 
spatial light modulator does not imprint phases larger than $3 \pi / 2$. Numerical simulations with other initial phase configurations, such as equally distributed phases along the four quadrants, have shown the same qualitative results, as we show in Sec. III.
[57] R. Fischer, I. Vidal, D. Gilboa, R. R. B. Correia, A. C. RibeiroTeixeira, S. D. Prado, J. Hickman, and Y. Silberberg, Phys. Rev. Lett. 115, 073901 (2015).

[58] H. Frostig, I. Vidal, R. Fischer, H. H. Sheinfux, and Y. Silberberg, Optica 7, 864 (2020). 